\title{
Arc-Disjoint Paths in Expander Digraphs
}

\author{
Tom Bohman* and Alan Frieze ${ }^{\dagger}$ \\ Department of Mathematical Sciences, \\ Carnegie Mellon University \\ Pittsburgh PA 15213.
}

\begin{abstract}
Given a digraph $D=(V, A)$ and a set of $\kappa$ pairs of vertices in $V$, we are interested in finding for each pair $\left(x_{i}, y_{i}\right)$, a directed path connecting $x_{i}$ to $y_{i}$, such that the set of $\kappa$ paths so found is arc-disjoint. For arbitrary graphs the problem is $\mathcal{N} \mathcal{P}$-complete, even for $\kappa=2$.

We present a polynomial time randomized algorithm for finding arc-disjoint paths in an $r$-regular expander digraph $D$. We show that if $D$ has sufficiently strong expansion properties and $r$ is sufficiently large then all sets of $\kappa=\Omega(n / \log n)$ pairs of vertices can be joined. This is within a constant factor of best possible.
\end{abstract}

\section{Introduction}

Given a graph/digraph $D=(V, A)$ with $n$ vertices and a set of $\kappa$ pairs of vertices in $V$, we are interested in finding, for each pair $\left(x_{i}, y_{i}\right)$, a directed path connecting $x_{i}$ to $y_{i}$, such that the set of $\kappa$ paths so found is edge/arc-disjoint. This is a classical problem in graph theory. See Frank [7] for a survey and Chapter 9.2 of the recent book on digraphs by Bang-Jensen and Gutin [2].

For undirected graphs, the related decision problem is in $\mathcal{P}$ for fixed $\kappa-$ Robertson and Seymour [22], but is $\mathcal{N} \mathcal{P}$-complete if $\kappa$ is part of the input. For digraphs the situation is seemingly much worse. Fortune, Hopcroft and Wylie [6] showed that the related decision problem is $\mathcal{N} \mathcal{P}$-complete, even when $\kappa=2$.

For undirected graphs there have been positive results in the case of expanders. Peleg and Upfal [21] presented a polynomial time algorithm for the case where $D$ is a (sufficiently strong) bounded degree expander graph and $\kappa \leq n^{\epsilon}$ for a small constant $\epsilon$ that depends on the expansion property of the graph. This result has been improved and extended by

\footnotetext{
${ }^{*}$ Supported in part by a grant from the NSA.

${ }^{\dagger}$ Supported in part by NSF grant CCR-9818411.
} 
Broder, Frieze, and Upfal [4, 5], Frieze [8, 9], Leighton and Rao [17] and Leighton, Rao and Srinivasan [18, 19]. In particular Frieze [9] showed that if $D$ has sufficiently strong edge expansion properties and $r$ is sufficiently large then all sets of $\kappa=\Omega(n / \log n)$ pairs of vertices can be joined. This is within a constant factor of a simple upper bound. The purpose of this paper is to extend this result to digraphs.

In this paper we discuss $r$-regular digraphs. A digraph $D$ is $r$-regular if every vertex has in-degree and out-degree $r$. Let $d_{\mu}$ be the median distance between pairs of vertices in the digraph $D$ which has $m$ arcs. Clearly, there exists a collection of $O\left(m / d_{\mu}\right)$ pairs of vertices that cannot be connected by arc-disjoint paths because such a collection of paths would require more arcs than all the arcs available. In the case of an $r$-regular expander, this absolute upper bound on $\kappa$ is $O(n / \log n)$ (assuming $r$ is independent of $n$ ). We show that if $D$ has sufficiently strong arc expansion properties and $r$ is sufficiently large then all sets of $\kappa=\Omega(n / \log n)$ pairs of vertices can be joined. This therefore, is within a constant factor of the optimum. The precise definition of "sufficiently strong" is given after the theorem.

Theorem 1 Let $D=(V, A)$ be an n-vertex, r-regular digraph. Suppose that $D$ is a sufficiently strong arc expander. Then there exist $\epsilon_{1}, \epsilon_{2}>0$ such that $D$ has the following property: For all sets of pairs of vertices $\left\{\left(x_{i}, y_{i}\right) \mid i=1, \ldots, \kappa\right\}$ satisfying:

(i) $\kappa=\left\lceil\epsilon_{1} r n / \log n\right\rceil$.

(ii) For each vertex $v,\left|\left\{i: x_{i}=v\right\}\right|,\left|\left\{i: y_{i}=v\right\}\right| \leq \epsilon_{2} r$.

There exist arc-disjoint paths in $D$, each of length $O(\log n)$, joining $x_{i}$ to $y_{i}$, for each $i=$ $1,2, \ldots, \kappa$. Furthermore, there is a polynomial time randomized algorithm for constructing these paths. The constants $\epsilon_{1}, \epsilon_{2}$ depend only on certain expansion parameters $\alpha, \beta, \gamma$ defined below. They do not depend on $n$ or $r$. (For example conditions (3) with $\epsilon=\alpha$, (4), (10) and (13) suffice).

Remark 1 The algorithm is similar to the algorithm of [9]. The difficulty in moving from graphs to digraphs has been with that part of the algorithm for graphs which was based on the rapid mixing of a random walk on expanders. Random walks on digraphs are not necessarily time reversible and the steady state can be hard to determine. We therefore abandoned this approach and replaced it with a different random choice of path (in part, this random choice for digraphs uses the multicommodity flow results of Leighton and Rao [16]).

It will be observed that this new algorithm can substitute for that given in [9].

Remark 2 If $D$ has sufficiently strong vertex expansion properties then we can take $\kappa=$ $\left\lceil\epsilon_{1} r n / \log _{r} n\right\rceil$ - see Remark 3 below.

\subsection{Preliminaries}

In this section we state the definitions for the expanders we work with here, make some preliminary observations about such expanders, and make precise the notion of 'sufficiently strong' expansion needed for Theorem 1 . We begin with some notational conventions. 
Let $D=(V, A)$ be a digraph and let $n=|V|$. For $S, T \subseteq V$ let $A_{D}^{+}(S, T)$ be the set of arcs with tail in $S$ and head in $T$; that is,

$$
A^{+}(S, T)=A_{D}^{+}(S, T)=\{(u, v) \in A \mid u \in S, v \in T\} \quad \text { and } \quad d^{+}(S, T)=\left|A^{+}(S, T)\right| .
$$

We define $A^{-}(S, T)$ simillarly (i.e $A^{-}(S, T)=A^{+}(T, S)$ ). We set

$$
A^{+}(S)=\{(u, v) \in A \mid u \in S, v \notin S\} \quad \text { and } \quad d^{+}(S)=\left|A^{+}(S)\right| .
$$

So, for example, we have $A^{+}(S)=A^{+}(S, V \backslash S)$. We define $A^{-}(S)$ and $d^{-}(S)$ analogously. Throughout the paper, when $\star$ is used as a subscript or superscript, it stands for + or -. We appreviate $A^{*}(\{v\}, T)$ to $A^{\star}(v, T)$. Thus, for $v \in V, d_{D}^{+}(v)$ and $d_{D}^{-}(v)$ denote the out-degree and in-degree of $v$ in $D$.

We now have the notation necessary to introduce expansion. We define expanders in terms of arc expansion (a weaker property than vertex expansion). For $S \subseteq V$ let $\Phi_{S}^{\star}=d^{\star}(S) /|S|$. The (arc-)expansion $\Phi=\Phi(D)$ of $D$ is defined by

$$
\Phi=\min _{\substack{S \subseteq V \\|S| \leq n / 2}} \min \left\{\Phi_{S}^{+}, \Phi_{S}^{-}\right\} .
$$

A digraph $D=(V, A)$ is a $\theta$-expander, if for every set $S \subseteq V,|S| \leq n / 2$, we have $d^{\star}(S) \geq \theta|S|$; in other words, $D$ is a $\theta$-expander if $\Phi(D) \geq \theta$. An $r$-regular digraph $D=(V, A)$ is called an $(\alpha, \beta, \gamma)$-expander if for every set $S \subseteq V$

$$
d^{\star}(S) \geq \begin{cases}(1-\alpha) r|S| & \text { if }|S| \leq \gamma n \\ \beta r|S| & \text { if } \gamma n<|S| \leq n / 2\end{cases}
$$

We naturally assume that $\beta<1-\alpha$. By "sufficiently strong" in Theorem 1 , we mean that $\beta, \gamma$ are arbitrary and $\alpha$ is sufficiently small; in particular, we assume that conditions (3) with $\epsilon=\alpha,(4),(10)$ and (13) hold. We also assume throughout that $r$ and $n$ are sufficiently large (but $r$ is not a function of $n$ ). We have made no real attempt to optimize constants.

We conclude this section with some preliminary observations about expanders. Since $\left|A^{\star}(S, S)\right|+d^{\star}(S)=r|S|$ we see that, putting $\operatorname{in}(S)=\left|A^{+}(S, S)\right|=\left|A^{-}(S, S)\right|$, in an $(\alpha, \beta, \gamma)$-expander

$$
\operatorname{in}(S) \leq \alpha r|S| \quad \text { when }|S| \leq \gamma n .
$$

In particular random regular digraphs are usually $(\alpha, \beta, \gamma)$-expanders. (See discussion in [4] for the corresponding notion in undirected graphs.) For a digraph $\Delta=\left(V^{\prime}, A^{\prime}\right)$ and a set $S \subseteq V^{\prime}$ we define its out-neighbor set $N_{\Delta}^{+}(S)$, as

$$
N_{\Delta}^{+}(S)=\left\{w \notin S: \exists v \in S \text { such that }(v, w) \in A^{\prime}\right\} .
$$

Similarly, the in-neighbor set of $S, N_{\Delta}^{-}(S)$ is given by

$$
N_{\Delta}^{-}(S)=\left\{w \notin S: \exists v \in S \text { such that }(w, v) \in A^{\prime}\right\} .
$$


Lemma 1 Suppose that $D=(V, A)$ is an $(\alpha, \beta, \gamma)$-expander and that $D^{\prime}=\left(V^{\prime}, A^{\prime}\right)$ is a sub-digraph of $D$ of expansion at least $\theta r$ where $\theta>\alpha$. Suppose $S \subseteq V^{\prime}$. If $|S| \leq \frac{\gamma \alpha}{\theta} n$ then

$$
\left|N_{D^{\prime}}^{\star}(S)\right| \geq \frac{\theta-\alpha}{\alpha}|S| .
$$

Proof: Suppose that $|S| \leq \frac{\gamma \alpha}{\theta} n$ and $T=N_{D^{\prime}}^{\star}(S)$ satisfies $|T|<\frac{\theta-\alpha}{\alpha}|S|$. Then

$$
|S \cup T| \leq\left(1+\frac{\theta-\alpha}{\alpha}\right)|S|=\frac{\theta}{\alpha}|S| \leq \gamma n .
$$

But, $S \cup T$ contains at least

$$
\theta r|S|>\theta r\left(1+\frac{\theta-\alpha}{\alpha}\right)^{-1}|S \cup T|=\alpha r|S \cup T|
$$

arcs, which contradicts (1).

\section{The algorithm}

The input to our algorithm is a sufficiently strong $(\alpha, \beta, \gamma)$-expander digraph $D$ and a set of pairs of vertices $\left\{\left(x_{i}, y_{i}\right) \mid i=1, \ldots, \kappa\right\}$ satisfying the premises of Theorem 1 . The output is a set of $\kappa$ arc-disjoint paths, $P_{1}, \ldots, P_{\kappa}$ such that $P_{i}$ connects $x_{i}$ to $y_{i}$.

The algorithm has three phases. We begin in Phase 0 by splitting our graph into 13 arc-disjoint expanders $D_{i}=\left(V, A_{i}\right), 1 \leq i \leq 13$. These graphs will be used for various purposes in Phases 1 and 2, and each path we construct will be a union of paths in the $D_{i}$ 's. Phase 1 consists mainly of applications of GeNPATHS, an algorithm that uses the expander property to naively connect pairs of vertices with paths of length $O(\log n)$ one at a time, deleting the arcs from a path as soon as it is used. Of course, this deletion of arcs may quickly destroy the expander property. To compensate for this problem, GeNPATHS 'shrinks' the expanders in which it finds paths. The real work of GeNPATHs is in keeping as many vertices as possible 'connected' to these shrinking expanders. Those pairs of vertices that are not connected with paths in Phase 1 (i.e. those pairs that contain a vertex whose connection with one of the 'shrinking expanders' is lost) are handled in Phase 2. There are $O\left(\frac{n}{\log ^{4} n}\right)$ such pairs. Loosely speaking, Phase 2 uses the multicommodity flow algorithm of Leighton and Rao to give a distribution on paths connecting the remaining pairs such that whp paths chosen at random with respect to this distribution are arc disjoint.

\subsection{Phase 0.}

We need an algorithm for splitting a $(\alpha, \beta, \gamma)$-expander digraph into 13 expander digraphs. Algorithms for splitting undirected graphs are given in [4] or [10]. They are easily adapted 
to digraphs and we outline an adaptation of the algorithm of [10] in Appendix A. The expansion requirements for this algorithm are

$$
\frac{r}{\log r} \geq 91 \epsilon^{-2} \text { and } \Phi \geq 65 \epsilon^{-2} \log 2 e r
$$

Since the arc-expansion of a $(\alpha, \beta, \gamma)$-expander is $\beta r$, we need

$$
\beta \geq 65 \epsilon^{-2} r^{-1} \log 2 e r .
$$

In the appendix we prove:

Theorem 2 Suppose that (2) holds and that $G$ is an $r$-regular $(\alpha, \beta, \gamma)$-expander, $r$ constant. Then there is a randomised polynomial time algorithm which with probability at least $1-\delta$ constructs $A_{1}, A_{2}, \ldots, A_{13}$ such that the arc-expansion $\Phi_{i}$ of $D_{i}=\left(V, A_{i}\right)$ satisfies

$$
\Phi_{i} \geq(1-\epsilon) \frac{\Phi}{13}-(\alpha+2 \epsilon) r
$$

for $i=1,2, \ldots, 13$.

This theorem is only useful if $\Phi$ is at least a constant multiple of $r$ and $\alpha$ is sufficiently small. This is the case discussed in this paper. The algorithm runs in $O\left(n^{2} \ln n \log \delta^{-1}\right)$ expected time.

We apply the algorithm of Theorem 2 with $\alpha=\epsilon$ and assume that

$$
\beta>156 \alpha
$$

Setting

$$
\beta_{0}=\frac{\beta}{13}-4 \alpha>8 \alpha>0
$$

each $D_{i}$ satisfies

$$
\begin{gathered}
\Phi_{i}=\Phi\left(D_{i}\right) \geq \beta_{0} r, \quad \text { and } \\
\beta_{0} r \leq d_{i}^{\star}(v)<r, \quad \text { for all } v \in V .
\end{gathered}
$$

\subsection{Phase 1.}

Phase 1 uses expanders $D_{1}$ through $D_{6}$. The centerpiece of Phase 1 is the algorithm GENPATHS that connects a large collection of random pairs of vertices in an expander with arc-disjoint paths. Since the pairs $x_{i}, y_{i}$ are arbitrary, GenPATHs cannot be applied to them directly; we must reduce the problem of connecting the $x_{i}$ 's to the $y_{i}$ 's to the problem of connecting random pairs.

In order to produce random pairs we introduce three random sets of $\kappa$ vertices: $\tilde{X}, \tilde{Y}$ and $Z$. In the Initialization step of Phase 1, a network-flow technique is used to find a collection 
of arc-disjoint paths $\mathcal{P}^{1}=\left\{P_{i}^{1}: i=1, \ldots, \kappa\right\}$ from $X=\left\{x_{1}, \ldots, x_{\kappa}\right\}$ to $\tilde{X}$ in expander $D_{1}$ such that the path $P_{i}^{1}$ starts at $x_{i}$ for $i=1, \ldots, \kappa$. It is important to note that we have no control over which element of $\tilde{X}$ is at the end of the path $P_{i}^{1}$ (but there will be one path ending at $x$ for each $x \in \tilde{X}$ ). This network-flow technique is also used in the Initialization step of Phase 1 to find a collection of arc-disjoint paths $\mathcal{P}^{6}=\left\{P_{i}^{6}: i=1, \ldots, \kappa\right\}$ from $\tilde{Y}$ to $Y=\left\{Y_{1}, \ldots, Y_{\kappa}\right\}$ in $D_{6}$ such that the endpoint of the path $P_{i}^{6}$ is $y_{i}$. After the Initialization step, we take a random ordering of $\tilde{X}: \tilde{X}=\left\{\tilde{x}_{1}, \ldots \tilde{x}_{\kappa}\right\}$. Furthermore, we order $\tilde{Y}$ so as to respect the pairing of $\tilde{X}$ and $\tilde{Y}$ that is inherited from the collections $\mathcal{P}^{1}$ and $\mathcal{P}^{6}$; that is, we set $\tilde{Y}=\left\{\tilde{y}_{1}, \ldots, \tilde{y}_{\kappa}\right\}$ so that if the endpoint of $P_{i}^{1}$ is $\tilde{x}_{j}$ then $\tilde{y}_{j}$ is the starting point of $P_{i}^{6}$. Thus, it remains to find a collection of arc-disjoint paths connecting the pairs $\left\{\left(\tilde{x}_{i}, \tilde{y}_{i}\right): i=1, \ldots, \kappa\right\}$. Unfortunately this sequence of pairs of vertices is not truly random. This is a consequence of the fact that the pairing between $\tilde{X}$ and $\tilde{Y}$ is determined by a deterministic process (i.e. knowledge of some of the pairs from this collection may bias the distribution on the unknown pairs). We overcome this problem by introducing the third random set $Z=\left\{z_{1}, \ldots, z_{\kappa}\right\}$. The sequences $\left\{\left(\tilde{x}_{i}, z_{i}\right): i=1, \ldots, \kappa\right\}$ and $\left\{\left(z_{i}, \tilde{y}_{i}\right): i=1, \ldots, \kappa\right\}$ are perfectly random sequences of pairs of vertices (so long as we view them separately). It remains to connect these two sequences of pairs of vertices with arc-disjoint paths. This is the work of the algorithm GeNPATHS.

The input to GenPaths is a pair of expanders, $D_{a}$ and $D_{b}$, and a collection of pairs of vertices $\left\{\left(v_{i}, u_{i}\right): i=1, \ldots, \kappa\right\}$ that is generated uniformly at random. The output of GenPaths is a collection of arc-disjoint paths from $v_{i}$ to $u_{i}$ for $i=1, \ldots, \kappa$ that use only the arcs from $D_{a}$ and $D_{b}$. We apply GenPaths twice. For the first application we set $D_{a}=D_{2}, D_{b}=D_{3}$ and $\left\{\left(v_{i}, u_{i}\right): i=1, \ldots, \kappa\right\}=\left\{\left(\tilde{x}_{i}, z_{i}\right): i=1, \ldots, \kappa\right\}$. In the second application we set $D_{a}=D_{4}, D_{b}=D_{5}$ and $\left\{\left(v_{i}, u_{i}\right): i=1, \ldots, \kappa\right\}=\left\{\left(z_{i}, \tilde{y}_{i}\right): i=1, \ldots, \kappa\right\}$. Now, the expander $D_{a}$ is used to connect the $v_{i}$ 's to the $u_{i}$ 's with short paths one at a time. In order to be sure that such paths exist we must be working with an expander. Therefore we delete some vertices in the course of the algorithm; in other words, this expander shrinks as the algorithm progresses. $D_{b}$ is used to keep as many vertices as possible connected to the 'shrinking expander' contained in $D_{a}$. We should note that these connections also require that $D_{b}$ be an expander. So, $D_{b}$ also 'shrinks' in the course of the algorithm. The subroutines Remove and ConNectBack are used by GenPaths.

\subsubsection{Initialization}

Let $\tilde{X}, \tilde{Y}$ be two randomly chosen $\kappa$-subsets of $V$. We begin by replacing the problem of finding paths from $x_{i}$ to $y_{i}$ by that of finding paths from $a_{i}$ to $b_{i}$, where $a_{i} \in \tilde{X}$ and $b_{i} \in \tilde{Y}$. Let $X$ denote the set $\left\{x_{1}, x_{2}, \ldots, x_{\kappa}\right\}$ and $Y=\left\{y_{1}, y_{2}, \ldots, y_{\kappa}\right\}$. We connect $X$ to $\tilde{X}$ via arc-disjoint paths in the digraph $D_{1}$ using a network flow. We construct our network as follows

- Each directed arc of $D_{1}$ gets capacity 1 .

- Each $v \in V$ becomes a source of capacity $\left|\left\{i: x_{i}=v\right\}\right|$ and each member of $\tilde{X}$ becomes a sink of capacity 1 . 
Then we find a flow from $X$ to $\tilde{X}$ that satisfies all demands. Since the maximum flow has integer values, it decomposes naturally into $|X|$ arc-disjoint paths (together perhaps with some cycles). We connect $Y$ to $\tilde{Y}$ by arc-disjoint paths in a similar manner using $D_{6}$

We now have a collection of arc-disjoint paths $\mathcal{P}^{1}=\left\{P_{i}^{1}: i=1, \ldots \kappa\right\}$ such that the starting point of $P_{i}^{1}$ is $x_{i}$ for $i=1, \ldots, \kappa$ and each member of $\tilde{X}$ is the endpoint of exactly one path from $\mathcal{P}^{1}$. Furthermore, we have a collection of arc-disjoint paths $\mathcal{P}^{6}=\left\{P_{i}^{6}: i=1, \ldots \kappa\right\}$ such that each member of $\tilde{Y}$ is the starting point of exactly one path from $\mathcal{P}^{6}$ and the end-point of path $P_{i}^{6}$ is $y_{i}$ for $i=1, \ldots, \kappa$.

\subsubsection{Algorithm GenPaths.}

The aim of GenPaths is to join $v_{i}$ and $u_{i}$ for $i=1,2, \ldots, \kappa$ by a short (i.e. of length $O(\log n))$ path in $D_{a}$. After constructing a path, we remove its arcs. It is important to ensure that short paths exist. Of course, this would not be a problem if we could ensure that $D_{a}$ remains an expander throughout. We have to be satisfied with identifying a dynamically changing large subgraph $\Delta_{a}=\left(V_{a}, F_{a}\right)$ of $D_{a}$ which is an expander. Initially $\Delta_{a}=D_{a}$, and $V_{a}$ loses vertices as GenPATHS progresses. We ensure that $\Delta_{a}$ remains an expander by keeping the degrees of vertices in the $\Delta_{a}$ close to their degree in $D_{a}$. This may involve deleting some (low degree) vertices after the construction of a path. We use the routine REMOVE to do this.

If the proposed start vertex $v$ of a walk on $\Delta_{a}$ does not lie in $V_{a}$ then we try to connect it back to $V_{a}$ by a path in $D_{b}$. The terminal endpoint of this walk is denoted by $v^{\prime}$. We use a subroutine ConnectBACK for this purpose. Similarly, the proposed end vertex $u$ might not lie in $V_{a}$. In this case we use ConNECTBACK to find a path from some $u^{\prime} \in V_{a}$ to $u$ in $D_{b}$. We do not expect to succeed all the time and our failures are kept in a set $L$ for treatment in Phase 2. The arcs in the paths generated by ConNECTBACK are deleted from $D_{b}$. Since ConnectBack requires that $D_{b}$ is an expander we will also be working with a second 'shrinking expander' $\Delta_{b}=\left(V_{b}, F_{b}\right)$ contained in $D_{b}$. This shrinking expander will also be maintained by use of the subroutine REMOVE.

In the end, the path from $v_{i}$ to $u_{i}$ will be a concatenation of up to three separate paths. There will always be a path $Q_{i}$ from $D_{a}$, and there may also be a short walk (or walks) from $D_{b}$ provided by ConNECTBACK (these are denoted $W_{i}^{C B \rightarrow}$ and $W_{i}^{C B \leftarrow}$, respectively). 


\section{Algorithm GenPaths}

2. begin

3. $\Delta_{i} \leftarrow D_{i}, i=a, b$.

4. for $i=1$ to $\kappa$ do

5. Execute REMOVE $\left(\Delta_{a}\right)$

6. Execute Connect Back $\left(V_{a}, v_{i}, \rightarrow, v_{i}^{\prime}, i, W_{i}^{C B \rightarrow}\right)$

7. Execute ConNect BACK $\left(V_{a}, u_{i}, \leftarrow, u_{i}^{\prime}, i, W_{i}^{C B \leftarrow}\right)$

8. if $i \notin L$ then

9. Construct a shortest path $Q_{i}$ from $v_{i}^{\prime}$ to $u_{i}^{\prime}$ in $\Delta_{a}$.

10. $\quad P_{i} \leftarrow\left(W_{i}^{C B \rightarrow}, Q_{i}, W_{i}^{C B \leftarrow}\right)$

11. $\Delta_{a} \leftarrow \Delta_{a} \backslash E\left(P_{i}\right)$

12. fi

13. od

14. end GenPaths

\subsubsection{Subroutine REMOVE}

The purpose of REMOVE is to delete vertices which might prevent a digraph from being an expander. In the course of GENPATHS we apply REMOVE to $\Delta_{a}$ and $\Delta_{b}$. In words, this simple algorithm iteratively removes those vertices whose in/out degree is less than the original in/out degree minus $\beta_{0} r / 2$. To be precise, at the end of the algorithm we have a graph $\Delta_{t}=\left(V_{t}, F_{t}\right)$ where $t \in\{a, b\}$ such that

$$
v \in V_{t} \text { implies } d_{\Delta_{t}}^{\star}(v) \geq d_{D_{t}}^{\star}(v)-\beta_{0} r / 2 \geq \beta_{0} r / 2 .
$$

The final inequality in (8) follows from (7). It follows immediately from (8) that for $S \subseteq V_{t}$ we have

$$
d_{\Delta_{t}}^{\star}(S) \geq d_{D_{t}}^{\star}(S)-\beta_{0} r|S| / 2 \geq\left(\Phi_{t}-\beta_{0} r / 2\right)|S| .
$$

This implies that, provided neither $V_{a}$ nor $V_{b}$ become empty (an issue which we take up in the next section), both $\Delta_{a}$ and $\Delta_{b}$ are expanders throughout Phase 1:

$$
\Phi_{\Delta_{t}} \geq \Phi_{t}-\beta_{0} r / 2 \geq \beta_{0} r / 2 \quad \text { for } t=a, b .
$$




\section{Algorithm Remove $\left(\Delta_{t}\right)$}

2. begin

3. $\quad B \leftarrow\left\{v \in V_{t}: d_{\Delta_{t}}^{+}(v)<d_{D_{t}}^{+}(v)-\beta_{0} r / 2\right.$ or $\left.d_{\Delta_{t}}^{-}(v)<d_{D_{t}}^{-}(v)-\beta_{0} r / 2\right\}$.

4. $\quad$ if $B \neq \emptyset$ then

5. $\quad A \leftarrow V_{t} \backslash B$

6. $\quad d \leftarrow \max _{v}\left\{\max \left\{d_{D_{t}}^{+}(v)-d_{\Delta_{t}}^{+}(v, A), d_{D_{t}}^{-}(v)-d_{\Delta_{t}}^{-}(v, A)\right\}: v \in A\right\}$.

7. while $d>\beta_{0} r / 2$ do

8. $\quad C \leftarrow\left\{w \in A: \max \left\{d_{D_{t}}^{+}(w)-d_{\Delta_{t}}^{+}(w, A), d_{D_{t}}^{-}(w)-d_{\Delta_{t}}^{-}(w, A)\right\} \geq \beta_{0} r / 2\right\}$

9. $\quad B \leftarrow B \cup C$

10. $A \leftarrow A \backslash C$

9. $\quad d \leftarrow \max _{v}\left\{\max \left\{d_{D_{t}}^{+}(v)-d_{\Delta_{t}}^{+}(v, A), d_{D_{t}}^{-}(v)-d_{\Delta_{t}}^{-}(v, A)\right\}: v \in A\right\}$.

10. od

11. $V_{t} \leftarrow A$

12. fi

13. end REMOVE

\subsubsection{Subroutine ConNECTBACK.}

The purpose of ConNECTBACK is to connect a vertex $z$ to $V_{a}$ by means of a short walk in $D_{b}$. The direction of this walk is determined by the input dir. If $\operatorname{dir}=\rightarrow$ then a path from $z$ to $V_{a}$ is required, and if dir $=\leftarrow$ then a path from $V_{a}$ to $z$ is needed. If $z \in V_{a}$ already then ConNectBACK does nothing but relabel $z$ as $z^{\prime}$. Since $\left|V \backslash V_{a}\right|$ can be of order $n$ (this is discussed below), we must maintain the expander property of $\Delta_{b}$ in order to find short connecting paths. Thus we apply Remove to $\Delta_{b}$ in the course of ConNeCtBACK. Now, those pairs that contain a vertex that lies in $V \backslash\left(V_{a} \cup V_{b}\right)$ are passed to Phase 2 in the set $L$. Thus, the long term success of ConNECTBACK hinges on keeping $V \backslash\left(V_{a} \cup V_{b}\right)$ small. In fact, this can be viewed as the key point in all of GenPaths.

We keep $V_{b}$ large by ensuring that the paths we use in $\Delta_{b}$ are spread out; in other words, we avoid using too many paths through any one vertex. This is achieved whp; recall that the pairs of vertices $\left\{\left(v_{i}, u_{i}\right): i=1, \ldots, \kappa\right\}$ that are the input to GeNPATHS are assumed to be generated uniformly at random. When a path is needed (i.e when $z \notin V_{a}$ ) ConNeCTBACK constructs a collection $\mathcal{W}^{\text {dir }}$ of walks in $\Delta_{b}$. This collection has the following properties.

1. If $\operatorname{dir}=\rightarrow$ then every walk in $\mathcal{W}^{\text {dir }}=\mathcal{W}^{\rightarrow}$ is a walk from a distinct vertex in $V_{b} \backslash V_{a}$ to $V_{a}$.

2. If dir $=\leftarrow$ then every walk in $\mathcal{W}^{\text {dir }}=\mathcal{W}^{\leftarrow}$ is a walk from $V_{a}$ to a distinct vertex in $V_{b} \backslash V_{a}$.

3. No path in $\mathcal{W}^{\text {dir }}$ is longer than $22 \log \log n$.

4. No vertex of $D_{b}$ lies on more than $240(\log \log n)^{2}$ paths. 
The set of start vertices of the walks in $\mathcal{W}^{\rightarrow}$ is denoted $S_{C B}$ and the set of terminal vertices of the walks in $\mathcal{W}^{\leftarrow}$ is denoted $S_{C B}^{\leftarrow}$. Clearly, $S_{C B}^{\text {dir }} \subseteq V_{b} \backslash V_{a}$. The collection of walks will have the additional property

$$
\text { 5. }\left|V \backslash\left(V_{a} \cup S_{C B}^{\mathrm{dir}}\right)\right| \leq \frac{n}{(\ln n)^{4}} .
$$

In words, condition 3 says that the paths in $\mathcal{W}^{\text {dir }}$ are short, condition 4 says that the paths are 'spread out' and condition 5 says that very few vertices are left out of the collection.

We emphasis that $\mathcal{W}^{\text {dir }}$ is constructed without use of any information about $z$. Therefore, $z$ (which was a random vertex to begin with) can be viewed as a vertex chosen uniformly at random after the collection $\mathcal{W}^{\text {dir }}$ is constructed. Heuristically, we can think of $\mathcal{W}^{\rightarrow}$ and $\mathcal{W}^{\leftarrow}$ as collections of connecting paths that are updated whenever $\Delta_{a}$ or $\Delta_{b}$ 'shrink,' but we only 'look' at these collections when we need them.

If $z \in S_{C B}^{\text {dir }}$ then we connect $z$ back to $V_{a}$ by way of the unique path in $\mathcal{W}^{\text {dir }}$ that begins at $z$ (if $\operatorname{dir}=\rightarrow$ ) or ends at $z$ (if $\operatorname{dir}=\leftarrow$ ). If $z$ does not lie in $S_{C B}^{\operatorname{dir}}$ we put $i$ into $L$, where $z=\tilde{x}_{i}$ or $\tilde{y}_{i}$. Arc disjoint paths for the pairs $\left(\tilde{x}_{i}, \tilde{y}_{i}\right), i \in L$ are found in Phase 2.

A network flow technique for the construction of the collection of paths $\mathcal{W}^{\text {dir }}$ follows from the proof of Lemma 3, which is given in Section 2.3.3 below.

1. subroutine CONNECTBACK $\left(V_{a}, z, \operatorname{dir}, z^{\prime}, i, W_{C B}\right)$

2. begin

3. $\quad$ if $z \in V_{a}$

4. $\quad$ then $z^{\prime} \leftarrow z$

5. else

6. $\quad$ Execute REMOVE $\left(\Delta_{b}\right)$

7. $\quad$ Contruct $\mathcal{W}^{\text {dir }}$ (see Lemma 3 for algorithm).

8. $\quad$ if $z \notin S_{C B}^{\mathrm{dir}}$

9. $\quad$ then $L \leftarrow L \cup\{i\}$

$10 . \quad$ else

11. $W_{C B} \leftarrow$ the unique path in $\mathcal{W}^{\text {dir }}$ with start/terminal vertex $z$

12. $z^{\prime} \leftarrow$ terminal/start vertex of $W_{C B}$

13. $\Delta_{b} \leftarrow \Delta_{b} \backslash W_{C B}$

14. $\quad$ fi

15. fi

16. end CONNECTBACK

\subsection{Analysis of Phase 1}

There are three facts concerning Phase 1 that remain to be shown: that the flow needed in the Initialization exists, that $V_{3}$ stays large and that at the end of Phase 1 whp we have $L=O\left(\frac{n}{(\log n)^{4}}\right)$. 


\subsubsection{Initialization}

In this subsection we show that if (6) holds and $r$ is sufficiently large then we can find arcdisjoint paths from $\left\{x_{1}, \ldots, x_{\kappa}\right\}$ to $\tilde{X}$ in $D_{1}$ and arc-disjoint paths from $\tilde{Y}$ to $\left\{y_{1}, \ldots, y_{\kappa}\right\}$ in $D_{6}$, for any choice of $x_{1}, \ldots, y_{\kappa}$ consistent with the premises of Theorem 1 , and every choice for $\tilde{X}, \tilde{Y}$. We assume that we have

$$
8 \alpha>\epsilon_{2}>r^{-1}
$$

For $S \subseteq V$, let

$$
\alpha(S)=\sum_{v \in S}\left|\left\{i: x_{i}=v\right\}\right| \quad \text { and } \quad \xi(S)=|S \cap \tilde{X}| .
$$

It follows from a theorem of Gale [12] (see Bondy and Murty [3] Theorem 11.8) that if

$$
d_{D_{1}}^{+}(S) \geq \xi(\bar{S})-\alpha(\bar{S}), \quad \forall S \subseteq V .
$$

then there exists a flow in the network defined on $D_{1}$ such that exactly one unit of flow travels through each vertex in $\tilde{X}$ and the amount of flow traveling through each vertex $v \in\left\{x_{1}, \ldots, x_{\kappa}\right\}$ is $\left|\left\{i: x_{i}=v\right\}\right|$. In other words, (11) implies a successful run of Phase 2.

Now, if $|S| \leq n / 2$ then, applying (5), (6) and (10), we have

$$
d_{D_{1}}^{+}(S) \geq|S| \Phi_{1} \geq|S| \beta_{0} r \geq 8 \alpha r|S| \geq \epsilon_{2} r|S| \geq \alpha(S)-\xi(S)=\xi(\bar{S})-\alpha(\bar{S}) .
$$

On the other hand, if $|S|>n / 2$ then we have

$$
d_{D_{1}}^{+}(S)=d_{D_{1}}^{-}(\bar{S}) \geq|\bar{S}| \Phi_{1} \geq|\bar{S}| \beta_{0} r \geq \epsilon_{2} r|\bar{S}| \geq \xi(\bar{S})-\alpha(\bar{S}) .
$$

Therefore, Phase 1 succeeds with respect to $X, \tilde{X}$. The same argument applies to $Y, \tilde{Y}$. To ensure these paths are of length $O(\log n)$ we can solve a minimum cost maximum flow problem as indicated in Kleinberg and Rubinfeld [13].

\subsubsection{On the size of $V_{a}$}

Lemma 2 Throughout GenPaths we have

$$
\left|V_{a}\right| \geq\left(1-\gamma_{0}\right) n
$$

where

$$
\gamma_{0}=\frac{\beta_{0} \gamma}{10}
$$

Proof: It follows from (9) that $\Delta_{a}$ is a $\left(\beta_{0} r / 2\right)$-expander throughout the execution of Phase 1. It follows from Lemma 1 that the diameter of $\Delta_{a}$ is always at most

$$
\tau=\left\lceil 2 \log _{1+\beta_{0} / 2} n+\frac{4 \gamma \alpha}{\log \beta_{0} r}\right\rceil .
$$


Indeed, consider breadth first search from some $v \in V_{a}$. Let $L_{t}, t \geq 0$ be the vertices at distance $t$ from $v$. Lemma 1 implies that the cardinalities of the $L_{t}$ grow at a rate at least $1+\beta_{0} / 2$ until they reach size $\left(2 \gamma \alpha / \beta_{0}\right) n$. The same will be true for breadth first search to a target vertex $w$. This accounts for the first term in (12). Once we have $L_{t}$ reaches $\left(2 \gamma \alpha / \beta_{0}\right) n$ we use that fact that going to the next level involves finding $\beta_{0} r\left|L_{t}\right| / 2$ "new arcs", at least until the size $n / 2$ is reached. This accounts for the second term in (12).

Thus the total number of arcs in the paths that are removed from $D_{a}$ is at most $\kappa \tau$. Let $B$ be the set of vertices that are removed from $\Delta_{a}$ in the course of Phase 1, and let $B_{1}$ be the set of vertices in $B$ incident with at least $\beta_{0} r / 4$ of the paths that are generated in $D_{a}$. We have

$$
\left|B_{1}\right| \leq \frac{8 \kappa \tau}{\beta_{0} r} \leq \frac{\gamma_{0} n}{2}
$$

provided

$$
\epsilon_{1} \leq \frac{\beta_{0}^{2} \gamma}{400} \log \left(1+\frac{\beta_{0}}{2}\right) .
$$

where $\epsilon_{1}$ is as in the statement of Theorem 1. Let $B_{2}=B \backslash B_{1}$ (i.e. those vertices removed from $\Delta_{a}$ that lie on less than $\beta_{0} r / 4$ of the paths generated in $D_{a}$ ).

Assume for the sake of contradiction that $\left|B_{2}\right|>\left|B_{1}\right|$. Let $B_{3}$ be the first $\left|B_{1}\right|$ vertices of $B_{2}$ to join $B$. Note that the vertices in $B_{3}$ have a large degree to $B_{1} \cup B_{3}$ (otherwise these vertices would remain in $V_{a}$ ). Applying (5) we have

$$
i n_{D_{a}}\left(B_{1} \cup B_{3}\right) \geq \frac{\beta_{0} r}{4}\left|B_{3}\right|=\frac{\beta_{0} r}{8}\left|B_{1} \cup B_{3}\right|>\alpha r\left|B_{1} \cup B_{3}\right| .
$$

This contradicts (1).

Therefore, $|B|=\left|B_{1}\right|+\left|B_{2}\right| \leq 2\left|B_{1}\right| \leq \gamma_{0} n$.

\subsubsection{Analysis of CONNECTBACK}

Of course, the first order of business here is to show how the collection of paths $\mathcal{W}^{\text {dir }}$ is generated.

Lemma 3 Suppose that $D=(V, A)$ is an $(\alpha, \beta, \gamma)$-expander and that $D^{\prime}=\left(V^{\prime}, A^{\prime}\right)$ is a sub-digraph of $D$ of expansion at least $\theta r$ where $\theta>6 \alpha$. Suppose that $S \subseteq V^{\prime}$ and that $|S| \geq\left(1-\frac{\gamma \alpha}{\theta}\right) n$ and let $T=V^{\prime} \backslash S$. Then there exists $T^{\star} \subseteq T$, such that $D^{\prime}$ contains a collection of walks $\mathcal{W}^{\text {dir }}=\left\{W_{v}: v \in T^{\star}\right\}$ such that

1. for $\operatorname{dir}=\rightarrow / \leftarrow, v$ is the start/terminal vertex of $W_{v}$ for all $v \in T^{\star}$,

2. the terminal/start vertex of each $W_{v}$ is in $S$,

3. each $W_{v}$ is of length at most $22 \log \log n$,

4. no vertex of $D^{\prime}$ lies on more than $240(\log \log n)^{2}$ paths, and 
5. $\left|T \backslash T^{\star}\right| \leq \frac{n}{(\log n)^{4}}$.

Proof: Assume without loss of generality that $\operatorname{dir}=\rightarrow$. For $i=1,2, \ldots$ let

$$
T_{i}=\left\{v \in T: \operatorname{dist}_{D^{\prime}}(v, S)=i\right\},
$$

and set $T_{0}=S$. Since $N_{D^{\prime}}^{+}\left(\cup_{k \geq i} T_{k}\right) \subseteq T_{i-1}$, it follows from Lemma 1 that we have

$$
\left|T_{i-1}\right| \geq \zeta\left|T_{i}\right| \quad \text { for } i \geq 1,
$$

where $\zeta=\frac{\theta-\alpha}{\alpha}>5$. Setting $i_{0}=\lceil 11 \log \log n\rceil$ and $\hat{T}=\bigcup_{i \geq i_{0}} T_{i}$ It follows from (14) that we have

$$
|\hat{T}| \leq \frac{n}{2(\log n)^{4}}
$$

Fix $1 \leq i<i_{0}$. We define a flow network $\mathcal{N}_{i}$. The vertex set of $\mathcal{N}_{i}$ is $\{s, t\} \cup \bigcup_{j=1}^{j_{0}}\left(C_{j} \cup C_{j}^{\prime}\right)$ where $C_{1}$ and $C_{1}^{\prime}$ are disjoint copies of $T_{i}$, for $2 \leq j \leq i, C_{j}$ and $C_{j}^{\prime}$ are disjoint copies of $\bigcup_{\ell \geq i+1-j} T_{\ell}$ and, for $i<j \leq j_{0}=2 i_{0}, C_{j}$ and $C_{j}^{\prime}$ are disjoint copies of $V^{\prime}$. The vertices $s$ and $t$ will be the source and sink, respectively, for the flow we introduce to $\mathcal{N}_{i}$. A vertex $v$ in $V^{\prime}$ may appear many times in the vertex set of $\mathcal{N}_{i} ;$ a copy of $v$ in $C_{j}$ is denoted $v_{j}$, and a copy of $v$ in $C_{j}^{\prime}$ is denoted $v_{j}^{\prime}$. For ease of notation, we let $\phi$ be the map that takes the vertices of $\mathcal{N}_{i}$ to their corresponding vertices in $V^{\prime}$. The arc-set of $\mathcal{N}_{i}$ is defined as follows. There is an arc from $s$ to each vertex of $C_{1}$. Each $v \in S$ gives rise to $\operatorname{arcs}\left(v_{j}^{\prime}, t\right), i<j \leq j_{0}$. If $v_{j}^{\prime} \in C_{j}^{\prime}$ and $w_{j+1} \in C_{j+1}$ are such that $(v, w)$ is an $\operatorname{arc}$ of $D^{\prime}$ then $\left(v_{j}^{\prime}, w_{j+1}\right)$ is an arc of $\mathcal{N}_{i}$. All arcs described so far have infinite capacity. In addition there are arcs $\left(v_{j}, v_{j}^{\prime}\right)$ of unit capacity defining a perfect matching between $C_{j}$ and $C_{j}^{\prime}$ for $1 \leq j \leq j_{0}$.

Claim $1 \mathcal{N}_{i}$ contains an $s-t$ flow of value at least $\left|T_{i}\right|-\frac{n}{(\log n)^{5}}$.

We first show the Lemma follows from Claim 1. The flow given by Claim 1 defines paths in $D^{\prime}$ from all but at most $\frac{n}{(\log n)^{5}}$ vertices of $T_{i}$ to $S$, each of length at most $j_{0}$. No vertex of $V^{\prime}$ can be on more than $j_{0}$ paths since each visit to $v$ uses a $\left(v_{j}, v_{j}^{\prime}\right)$ arc for some $j$. Repeating this construction for $i=1,2, \ldots, i_{0}$ we find paths for all but a set $\tilde{T}$ of at most $i_{0} \frac{n}{(\log n)^{5}} \leq \frac{n}{2(\log n)^{4}}$ vertices and no vertex can be on more than $i_{0} j_{0}$ paths. Putting $T^{\star}=T \backslash(\hat{T} \cup \tilde{T})$ and using (15) gives us the lemma.

It only remains to prove Claim 1 . Of course, we do this via the max-flow min-cut theorem. Consider a cut $Z \dot{\cup} \bar{Z}$ of $\mathcal{N}_{i}$ where the vertex set $Z$ contains $s$ but not $t$. Let $A_{j}=Z \cap C_{j}, B_{j}=C_{j} \backslash A_{j}, A_{j}^{\prime}=Z \cap C_{j}^{\prime}$ and $B_{j}^{\prime}=C_{j}^{\prime} \backslash A_{j}^{\prime}$ for $j=1,2, \ldots, j_{0}$. Assume for the sake of contradiction that the capacity of this cut is less than $\left|C_{1}\right|-\frac{n}{(\log n)^{5}}$. It follows from this assumption that the cut contains no infinite capacity arcs and therefore

$$
A_{1}=C_{1}, \quad \phi\left(A_{j+1}\right) \supseteq N_{D^{\prime}}^{+}\left(\phi\left(A_{j}^{\prime}\right)\right) \text { for all } j, \quad \text { and } \quad \phi\left(A_{j}^{\prime}\right) \cap S=\emptyset \text { for all } j .
$$


The capacity of the cut is

$$
\left|B_{1}^{\prime}\right|+\sum_{j=2}^{j_{0}}\left|\phi\left(A_{j}\right) \cap \phi\left(B_{j}^{\prime}\right)\right|
$$

The third condition of (16) implies that for all $j,\left|A_{j}^{\prime}\right|<\frac{\gamma \alpha}{\theta} n$. It then follows from Lemma 1 and the second condition of (16) that we have $\left|A_{j+1}\right| \geq \zeta\left|A_{j}^{\prime}\right|$. This implies that for all $j$ we have either

$$
\left|A_{j+1}^{\prime}\right| \geq \frac{1}{2}\left|A_{j+1}\right| \geq \frac{\zeta}{2}\left|A_{j}^{\prime}\right|
$$

or

$$
\left|\phi\left(A_{j+1}\right) \cap \phi\left(B_{j+1}^{\prime}\right)\right| \geq \frac{1}{2}\left|A_{j+1}\right| \geq \frac{\zeta}{2}\left|A_{j}^{\prime}\right| .
$$

Now, if $\left|A_{1}^{\prime}\right| \leq \frac{n}{(\log n)^{5}}$ then $\left|B_{1}^{\prime}\right| \geq\left|C_{1}\right|-\frac{n}{(\log n)^{5}}$, which contradicts our initial assumption. On the other hand, if $\left|A_{1}^{\prime}\right|>\frac{n}{(\log n)^{5}}$ then, since $\left(\frac{\zeta}{2}\right)^{j_{0}-1}>(\log n)^{5}$, condition (18) cannot always hold. Let $j_{1} \geq 1$ be the first $j$ for which (19) holds. We have

$$
\left|\phi\left(A_{j_{1}+1}\right) \cap \phi\left(B_{j_{1}+1}^{\prime}\right)\right| \geq(\zeta / 2)^{j_{1}}\left|A_{1}^{\prime}\right| \geq\left|A_{1}^{\prime}\right| .
$$

The capacity of the cut is at least $\left|B_{1}^{\prime}\right|+\left|A_{1}^{\prime}\right|=\left|C_{1}\right|$. This is a contradiction.

To get the collection of paths needed for ConNeCTBACK we apply Lemma 3 with $D^{\prime}=\Delta_{b}, V^{\prime}=V_{b}, S=V_{a} \cap V_{b}$. So, for example, we have $S_{C B}^{\text {dir }}=T^{\star}$. It remains to show that whp we have $|L| \leq \frac{n}{(\log n)^{4}}$.

Note that Lemma 3 can only be applied if $V_{a} \cap V_{b}$ is large. However, by applying the proof of Lemma 2 and the fact that the paths generated by ConNECTBACK are short (length at most $22 \log \log n$ ) we see that $\left|V_{b}\right|=n-O(n \log \log n / \log n)$ ) throughout and this is sufficient. However, we shall see that $V_{b}$ is whp larger than this. This is where we use the fact that the paths in $\mathcal{W}^{\text {dir }}$ are spread out (i.e. the fact that there are at most $240(\log \log n)^{2}$ paths in $\mathcal{W}^{\text {dir }}$ through any one vertex $)$.

It follows from this fact that the probability that that an arbitrary vertex $w$ is on the path $W_{i}^{C B \text { dir }}$ at most $240(\log \log n)^{2} / n$. It follows that we have

$$
\begin{aligned}
\operatorname{Pr}\left(\left|\left\{i: w \in W_{i}^{C B \rightarrow}\right\}\right|+\right. & \left.\left|\left\{i: w \in W_{i}^{C B \leftarrow}\right\}\right| \geq 20\right) \\
& \leq \operatorname{Pr}\left(B\left(2 \kappa, 240(\log \log n)^{2} / n\right) \geq 20\right) \\
& \leq\left(\begin{array}{c}
2 \kappa \\
20
\end{array}\right)\left(\frac{240(\log \log n)^{2}}{n}\right)^{20} \\
& =o\left((\log n)^{-19}\right) .
\end{aligned}
$$

Let $B$ be the set of vertices which are removed from $\Delta_{b}$ by applications of REMOVE. Let $X_{1}$ be the set of vertices in $B$ that are on at least 20 of the paths $W_{i}^{C B \rightarrow}, W_{i}^{C B \leftarrow}$. Note 
that $X_{1}$ contains the set $B_{1}$ introduced in the proof of Lemma $2\left(B_{1}\right.$ is the collection of vertices taken out of $V_{b}$ by REMOVE that are on many of the paths). It follows from (20) and Markov's inequality that whp we have $\left|X_{1}\right| \leq n /(\log n)^{18}$. Now, $B$ (which contains $V \backslash V_{b}$ at every step) consists of $B_{1}$ together with extra vertices deleted by REMOVE. In total this will be at most $2\left|B_{1}\right|$ vertices removed by the argument of Lemma 2, following (13). Thus, $|B| \leq 2\left|B_{1}\right| \leq 2\left|X_{1}\right|$. Therefore

$$
|B| \leq \frac{n}{(\log n)^{18}}
$$

whp.

Now, a failure (i.e. the index $i$ joining the set $L$ ) can occur in one of two ways. On one hand we have a failure if either $v_{i}$ or $u_{i}$ does not lie in $V_{a} \cup V_{b}$, and on the other hand a failure results when $u_{i} \in V_{b} \backslash\left(V_{a} \cup S_{\overrightarrow{C B}}\right)$ or $v_{i} \in V_{b} \backslash\left(V_{a} \cup S_{C B}^{\leftarrow}\right)$. It follows from (21) that the total number of failures of the first type is whp at most $\frac{n}{(\log n)^{18}}$. For failures of the second type we note that $v_{1}, \ldots, v_{\kappa}$ form a random sequence of size $o(n)$. The probability that a particular vertex gives a failure of the second kind is at most

$$
\frac{\left|S_{C B}^{\mathrm{dir}}\right|}{n}=O\left(\frac{1}{(\log n)^{4}}\right) .
$$

Therefore, whp the total number of failures of the second kind is $O\left(n /(\log n)^{4}\right)$.

Remark 3 Suppose $G$ has the following vertex expansion property for small sets: $S \subseteq$ $V,|S| \leq \frac{\gamma}{r} n$ implies that $\left|N_{D}^{\star}(S)\right| \geq(1-\alpha) r|S|$. The algorithm of Lemma \& can be modified to split $D$ so that each subgraph $D_{i}$ and small $S$ satisfies $\left|N_{D_{i}}^{\star}(S)\right| \geq \frac{1-30 \alpha}{13} r|S|$. Then the shortest paths in $\Delta_{a}$ will be of length $O\left(\log _{r} n\right)$ and the claim in Remark 2 will follow.

\subsection{Phase 2.}

The set of pairs $\left\{\left(\tilde{x}_{i}, \tilde{y}_{i}\right): i \in L\right\}$ have not yet been connected by paths. We have seen that whp the number of such pairs, $|L|$, is at most $O\left(n /(\log n)^{4}\right)$. These pairs are dealt with by the algorithm described below which uses digraphs $D_{7}-D_{13}$.

The heart of the algorithm is a randomized method (based on a multicommodity flow result of Leighton and Rao [16]) for connecting pairs of vertices with arc disjoint paths which works whp when the collection of pairs is generated uniformly at random. So, as in Phase 1, some preliminary steps must be taken in order to reduce the problem of connecting an arbitrary set of pairs of vertices with arc-disjoint paths to the problem of connecting a random collection of pairs with arc-disjoint paths. We proceed directly to a description of the algorithm. Let $m=|L|$ and $\lambda=\lceil\log n\rceil$.

We begin by 'amplifying' each start vertex $\tilde{x}_{i}, i \in L$ and each end vertex $\tilde{y}_{i}, i \in L$ to a collection of $\lambda$ vertices. This process occurs in steps (a) and (b).

(a) In this step, we choose a collection of vertices $w_{j}, 1 \leq j \leq 2 m$, and a collection of sets of vertices $W_{j}, 1 \leq j \leq 2 m$, such that for $1 \leq j \leq 2 m$, 
(i) $w_{j} \in W_{j}$,

(ii) $\left|W_{j}\right|=\lambda$,

(iii) The sets $W_{j}$ are pairwise disjoint, and

(iv) $D_{9}$ contains an arborescence with vertex set $W_{j}$ and root $w_{j}$. For $1 \leq j \leq m$ this arborescence is directed away from the root and for $m+1 \leq j \leq 2 m$ this arborescence is directed toward the root.

Following [17], we find these arborescences by partitioning large arborescences of $D_{\mathbf{9}}$. We begin with a rooted spanning arborescence $T$ with the property that all arcs are directed away from the root. We generate $W_{1}, \ldots, W_{m}$ greedily from $D_{9}$, removing an arborescence from $T$ once it is used. Of course, this process will divide $T$ into a number of components. However, since the maximum degree of $D_{9}$ is $r$, the number of components produced in this process is at most $r \lambda m=O\left(\frac{n r}{(\log n)^{3}}\right)$. Since any tree having at least $\lambda$ vertices contains a subtree having exactly $\lambda$ vertices, we will always be able to find the needed arborescences.

We then apply Remove to $D_{9}$ less the vertex set $\cup_{i=1}^{m} W_{i}$ to produce an expander $D_{9}^{\prime}$. It follows from the proof of Lemma 2 that $D_{9}^{\prime}$ has $n(1-o(1))$ vertices. We repeat the process described above (this time using $D_{9}^{\prime}$ and an arborescense directed towards the roots) to produce $W_{m+1}, \ldots, W_{2 m}$.

(b) Let $S_{X}=\left\{\tilde{x}_{i}: i \in L\right\}$ and $S_{Y}=\left\{\tilde{y}_{i}: i \in L\right\}$ denote the sets of vertices that need to be joined. Use a network flow algorithm (analogous to what is given in the Initialization step of Phase 1) in $D_{7}$ to connect in an arbitrary manner the vertices of $S_{X}$ to to $W_{X}=\left\{w_{1}, \ldots, w_{m}\right\}$ by $m$ arc disjoint paths. Using the same network flow algorithm in $D_{8}$, connect in an arbitrary manner the vertices of $W_{Y}=\left\{w_{m+1}, \ldots, w_{2 m}\right\}$ to $S_{Y}$ by $m$ arc disjoint paths. The expansion properties of $D_{7}$ and $D_{8}$ ensure that such paths always exist (as we saw in the Initialization step of Phase 1).

Let $\hat{x}_{k}$ (resp. $\hat{y}_{k}$ ) denote the vertex in $W_{X}$ that was connected to the end-point $\tilde{x}_{k}$ (resp. $\tilde{y}_{k}$ ). Our problem is now to find arc-disjoint paths joining $\hat{x}_{k}$ to $\hat{y}_{k}$ for $1 \leq k \leq m$. If $w_{t}$ has been renamed as $\hat{x}_{k}$ (resp. $\hat{y}_{k}$ ) then rename the elements of $W_{t}$ as $\hat{x}_{k, \ell}$ (resp. $\left.\hat{y}_{k, \ell}\right) 1 \leq \ell \leq \lambda$.

(c) Choose $\xi_{j}, 1 \leq j \leq \lambda m$, and $\eta_{j}, 1 \leq j \leq \lambda m$, uniformly at random from $V$. Using a network flow algorithm (as in (b)) connect $\left\{\hat{x}_{k, \ell}: 1 \leq k \leq m, 1 \leq \ell \leq \lambda\right\}$ to $\left\{\xi_{j}: 1 \leq j \leq \lambda m\right\}$ by arc-disjoint paths in $D_{10}$. Similarly, connect $\left\{\eta_{j}: 1 \leq j \leq \lambda m\right\}$ to $\left\{\hat{y}_{k, \ell}: 1 \leq k \leq m, 1 \leq \ell \leq \lambda\right\}$ by arc-disjoint paths in $D_{13}$. Rename the other endpoint of the path starting at $\hat{x}_{k, \ell}$ (resp. ending at $\left.\hat{y}_{k, \ell}\right)$ as $x_{k, \ell}^{*}$ (resp. $y_{k, \ell}^{*}$ ).

(d) Choose $z_{k, \ell}^{*}, 1 \leq k \leq m, 1 \leq \ell \leq \lambda$ uniformly at random, with replacement. Now, it is important to note that the pairs $x_{k, \ell}^{*}, z_{k, \ell}^{*}$ and the pairs $z_{k, \ell}^{*}, y_{k, \ell}^{*}$ are (when viewed separately) perfectly random. We are using the same 'trick' that we used in Phase 1 for replacing the problem of connecting arbitrary pairs to the problem of connecting random pairs. 
The paths between pairs of the form $x_{k, \ell}^{*}, z_{k, \ell}^{*}$ and between pairs of the form $z_{k, \ell}^{*}, y_{k, \ell}^{*}$ are generated at random. Using the multicommodity flow algorithm of Leighton and Rao [16], find a collection of paths $P_{u, v ; \theta}, u \neq v \in V, 1 \leq \theta \leq \nu_{u, v}$ where each $P_{u, v ; \theta}$ is a path in $D_{12}$ from $u$ to $v$. These are the flow paths given by Theorem 18 in [16] $\left(\nu_{u, v}\right.$ is simply the number of flow paths we have for the pairs $\left.u, v\right)$. Let $\mathcal{P}_{u, v}=\left\{P_{u, v ; \theta}: 1 \leq \theta \leq \nu_{u, v}\right\}$. For each $P_{u, v ; \theta}$ we will have a flow value $f_{u, v ; \theta}>0$ and we let $F_{u, v}=\sum_{\theta=1}^{\nu_{u, v}} f_{u, v ; \theta}$. The properties we require are that

(P1) For all arcs $e$ of $D_{10}$,

$$
\sum_{(u, v, \theta): e \in P_{u, v ; \theta}} f_{u, v ; \theta} \leq 1
$$

(P2)

$$
F_{u, v} \geq \frac{c_{2}}{n \log n}
$$

for some absolute constant $c_{2}>0$.

(P3) The length of each path $P_{u, v ; \theta}$ is at most $\lambda_{1}=c_{1} \log _{r} n$ for some absolute constant $c_{1}>0$.

For $u, v \in V$ let $P_{u, v}$ be the probability distribution over $\mathcal{P}_{u, v}$ where $P_{u, v}\left(P_{u, v ; \theta}\right)=$ $f(u, v, \theta) / F(u, v)$. Then for each $k, \ell$ choose $W_{k, \ell}^{\prime}$ randomly from $\mathcal{P}_{x_{k, \ell}^{*}, z_{k, \ell}^{*}}$ using the distribution $P_{x_{k, \ell}^{*}, z_{k, \ell}^{*}}$ to select the path.

Let $B_{k}^{\prime}$ denote the bundle of paths $\left\{W_{k, \ell}^{\prime}, 1 \leq \ell \leq \lambda\right\}$. Carry out the same construction in $D_{13}$ and construct a bundles of paths $B_{k}^{\prime \prime}=\left\{W_{k, \ell}^{\prime \prime}, 1 \leq \ell \leq \lambda\right\}$ where $W_{k, \ell}^{\prime \prime}$ is a path from $z_{k, \ell}^{*}$ to $y_{k, \ell}^{*}$.

Let $\pi_{0}=\max _{e} \operatorname{Pr}(e \in P)$ where $P$ is a path chosen by (i) randomly choosing endpoints $u, v$ and then (ii) choosing $P \in \mathcal{P}_{u, v}$ according to the distribution $P_{u, v}$. We have

$$
\pi_{0}=\max _{e} \sum_{P_{u, v ; \theta} \ni e} \frac{1}{n^{2}} \cdot \frac{f(u, v, \theta)}{F(u, v)} \leq \frac{1}{n^{2}} \frac{n \log n}{c_{2}}=\frac{\log n}{c_{2} n}
$$

We say that $W_{k, \ell}^{\prime}$ is $b a d$ if there exists $k^{\prime} \neq k$ such that $W_{k, \ell}^{\prime}$ shares an arc with a walk in a bundle $B_{k^{\prime}}^{\prime}$.

Now, suppose the bundles in the set $\left\{B_{j}: j \neq k\right\}$ are fixed. The collection of paths involved in these bundles gives at most $m \lambda \lambda_{1}$ arcs. Thus, the probability that $W_{k, l}$ is bad, conditioning on what happens outside the bundle $B_{k}$, is at most

$$
\pi_{0} m \lambda \lambda_{1}=O\left(\frac{1}{\log n}\right)
$$

We say that index $k$ is bad if either $B_{k}^{\prime}$ or $B_{k}^{\prime \prime}$ contain more than $\lambda / 3$ bad walks. If index $k$ is not bad then we can find a walk from $x_{k, \ell}^{*}$ to $y_{k, \ell}^{*}$ through $x_{k, \ell}^{*}$ for some $\ell$ which is arc disjoint from all other walks. This gives a walk

$$
x_{k}-\tilde{x}_{k}-\hat{x}_{k}-\hat{x}_{k, \ell}-x_{k, \ell}^{*}-x_{k, \ell}^{*}-y_{k, \ell}^{*}-\hat{y}_{k, \ell}-\hat{y}_{k}-\tilde{y}_{k}-y_{k},
$$


which is arc-disjoint from all other such walks.

The probability that index $k$ is bad is at most

$$
2 \operatorname{Pr}\left(B\left(\lambda, O\left((\log n)^{-1}\right) \geq \lambda / 3\right)=O\left(n^{-2}\right) .\right.
$$

So with probability $1-0(1)$ there are no bad indices.

\section{References}

[1] N. Alon and J.H. Spencer, The Probabilistic Method, Wiley, 1992.

[2] J. Bang-jensen and G. Gutin, Digraphs: Theory, Algorithms and Applications, Springer-Verlag, London 2001.

[3] J. A. Bondy and U. S. R. Murty, Graph Theory with Applications, North-Holland 1976.

[4] A. Z. Broder, A. M. Frieze, and E. Upfal, Existence and construction of edge disjoint paths on expander graphs, SIAM Journal on Computing 23 (1994) 976-989.

[5] A. Z. Broder, A. M. Frieze, and E. Upfal, Existence and construction of edge low congestion paths on expander graphs, Random Structures and Algorithms 14 (1999) 87-109.

[6] S. Fortune, J.E. Hopcroft and J. Wyllie, The directed subgraph homeomorphism problem, Theoretical Computer Science 10 (1980) 111-121.

[7] A. Frank, Disjoint paths in rectilinear grids, Combinatorica 2, (1982) 361-371.

[8] A.M. Frieze, Disjoint Paths in Expander Graphs via Random Walks: a Short Survey, Proceedings of Random '98, Lecture Notes in Computer Science 1518 (1998) Springer, 1-14.

[9] A.M. Frieze, Edge disjoint paths in expander graphs, Proceedings of the 11th Annual ACM-SIAM Symposium on Discrete Algorithms (2000) 717-725, (to appear in SIAM Journal on Computing).

[10] A.M.Frieze and M.Molloy, Splitting an expander graph, Journal of Algorithms 33 (1999) 166-172.

[11] A.M. Frieze and L. Zhao, Edge disjoint paths in random regular graphs, Proceedings of the 10th Annual ACM-SIAM Symposium on Discrete Algorithms (1999) 291-299 
[12] D. Gale, A theorem on flows in networks, Pacific Journal of Mathematics 7 (1957) 1073-1082.

[13] J. Kleinberg and R. Rubinfeld, Short paths in expander graphs, Proceedings of the 37th Annual IEEE Symposium on Foundations of Computer Science, (1996) 86-95.

[14] J. Kleinberg and E. Tardos, Approximations for the disjoint paths problem in high diameter planar networks, Proceedings of the 27'th Annual ACM Symposium on Theory of Computing, (1995) 26-35.

[15] D.E. Knuth, The art of computer programming, Volume 1, Fundamental Algorithms, Addison-Wesley, 1968.

[16] T. Leighton and S. Rao, Multicommodity max-flow min-cut theorems and their use in designing approximation algorithms, Journal of the Association for Computing Machinery 46 (1999) 787-832.

[17] T. Leighton and S. Rao, Circuit switching: a multicommodity flow based approach, Proceedings of a Workshop on Randomized Parallel Computing 1996.

[18] T. Leighton, S.Rao and A.Srinivasan, Multi-commodity flow and circuit switching, Proceedings of the Hawaii International Conference on System Sciences, 1998.

[19] T. Leighton, S. Rao and A. Srinivasan, New algorithmic aspects of the local lemma with applications to partitioning and routing, Proceedings of the 10th Annual ACMSIAM Symposium on Discrete Algorithms (1999) 643-652..

[20] A. Lubotsky, R. Phillips, and P. Sarnak, Ramanujan graphs, Combinatorica 8 (1988) 261-277.

[21] D. Peleg and E. Upfal, Constructing disjoint paths on expander graphs, Combinatorica 9, (1989) 289-313.

[22] N. Robertson and P. D. Seymour, Graph minors-XIII: The disjoint paths problem, to appear.

[23] A. Sinclair and M. Jerrum, Approximate counting, uniform generation, and rapidly mixing Markov chains, Information and Computation 82 (1989) 93-133.

[24] D. Wagner and K. Weihe, A linear time algorithm for edge-disjoint paths in planar graphs, Proceedings of the First European Symposium on Algorithms (ESA '93) Lecture Notes in Computer Science 726, Springer-Verlag (1992) 384-395.

[25] X. Zhou, S. Tamura and T. Nishizeki, Finding edge-disjoint paths in partial $k$-trees, Algorithmica 26 (2000) 3-30. 


\section{Appendix}

\section{A Splitting an expander digraph}

We prove two results on splitting $D$ into $D_{1} \cup \cdots \cup D_{k}$ where $D_{i}=\left(V, A_{i}\right)$. One is nonconstructive and shows what might be achieved. The second is constructive and uses the first. The split produced by the second is not as good as indicated by the first result. We use a subscript $i$ to denote graph-theoretic constructs related to $D_{i}$. Thus $d_{i}^{+}(v)$ is the out-degree of $v$ in $D_{i}$. Left unsubscripted, such things refer to $D$. Thus $d^{-}(v)=r$.

In Section B we prove

Theorem 3 Let $k \geq 2$ be a positive integer and let $\epsilon>0$ be a small positive real number. Suppose that

$$
\frac{r}{\ln r} \geq 7 k \epsilon^{-2} \text { and } \Phi \geq 5 \epsilon^{-2} k \ln 2 e r .
$$

Then there exists a partition $A=A_{1} \cup A_{2} \cup \cdots \cup A_{k}$ such that for $1 \leq i \leq k$

(a)

$$
\Phi_{i} \geq(1-\epsilon) \frac{\Phi}{k}
$$

(b)

$$
(1-\epsilon) \frac{r}{k} \leq \delta^{*}\left(D_{i}\right) \leq \Delta^{*}\left(D_{i}\right) \leq(1+\epsilon) \frac{r}{k} .
$$

We use this in the proof of

Theorem 4 Suppose that the conditions of Theorem 3 hold, and suppose further that $D$ is an $(\alpha, \beta, \gamma)$-expander. Then there is a randomised polynomial time algorithm

$\left(O\left(n^{2} \ln n \ln \delta^{-1}\right)\right)$ which with probability at least $1-\delta$ constructs

$A_{1}, A_{2}, \ldots, A_{k}$ such that

$$
\Phi_{i} \geq(1-\epsilon) \frac{\Phi}{k}-(\alpha+\epsilon) r
$$

for $i=1,2, \ldots, k$.

This theorem is only useful if $\Phi \geq c r$ for some $c$ satisfying $c \gg \alpha$. For random $r$-regular graphs we can take $\gamma$ to be a small constant and $\alpha=O\left(\gamma+\frac{1}{\sqrt{r}}\right)$.

Note that there is not enough time to verify that the algorithm succeeds. Instead, we assume it has and repeat the split if we fail to find the required paths. 


\section{B Existence Result}

We prove Theorem 3. We will use the general version of the Lovász Local Lemma. For each $a \in A$ we randomly choose an integer $i \in[k]$ and then place $a$ in $A_{i}$. We must show that there is a positive probability of choosing a partition which satisfies the conditions of the theorem.

We define the following bad events: First let $G=(V, E)$ be the $2 r$-regular (multi-)graph obtained by ignoring orientation in $D$. If $S \subseteq V$ then $G_{S}[V]=\left(S, E_{S}\right)$ is the subgraph of $G$ induced by $S$. We say that $S$ is connected if $D_{S}$ is.

(a) For $v \in V, i \in[k]$ and $* \in\{+,-\}, A_{v, i, *}=A_{\{v\}, i, *}$ is the event that

$$
d_{i}^{*}(v) \notin[(1-\epsilon) r / k,(1+\epsilon) r / k] .
$$

(b) For $S \subseteq V, 2 \leq|S| \leq n / 2, S$ connected, $i \in[k]$ and $* \in\{+,-\}, A_{S, i, *}$ is the event that

$$
\left|d_{i}^{*}(S)\right|<(1-\epsilon)\left|d^{*}(S)\right| / k
$$

In showing that $\Phi_{i}$ is sufficiently large we can restrict our attention to $S$ for which $S$ is connected. Indeed, for $S \subset V$ let $C_{1}, C_{2}, \ldots, C_{t}$ be the components of $G[S]$. Then for $* \in\{+,-\}$,

$$
\Phi_{S, i}^{*} \geq \min _{1 \leq s \leq t} \frac{d_{i}^{*}\left(C_{s}\right)}{\left|C_{s}\right|} .
$$

Claim 2 For $v \in V$ there are at most $(2 e r)^{s-1}$ sets $S$ such that $(i) v \in S$, (ii) $|S|=s$ and (iii) $S$ is connected.

Proof of Claim 2 The number of such sets is bounded by the number of distinct $s$-vertex trees which are rooted at $v$. This in turn is bounded by the number of distinct $2 r$-ary rooted trees with $s$ vertices. This is equal to $\left(\begin{array}{c}2 r s \\ s\end{array}\right) /((2 r-1) s+1)$, see Knuth [15].

End of proof of Claim 2

The Chernoff bounds for the tails of the binomial distribution $B(n, p)$ that we use are

$$
\begin{aligned}
& \operatorname{Pr}(B(n, p) \geq(1+\epsilon) n p) \leq e^{-\epsilon^{2} n p / 3} \\
& \operatorname{Pr}(B(n, p) \leq(1-\epsilon) n p) \leq e^{-\epsilon^{2} n p / 2}
\end{aligned}
$$

where $0 \leq \epsilon \leq 1$.

Using them we obtain,

$$
\operatorname{Pr}\left(A_{v, i, *}\right) \leq 2 e^{-\epsilon^{2} r /(3 k)} \leq 2 e^{-(7 \ln r) / 3}<\frac{1}{r^{2}}
$$

and

$$
\operatorname{Pr}\left(A_{S, i, *}\right) \leq \exp \left\{-\frac{\epsilon^{2} d^{*}(S)}{2 k}\right\} \leq e^{-2|S| \ln r}=\frac{1}{r^{2|S|}}
$$


Now, for $S \subseteq V, 1 \leq|S| \leq n / 2$ and $S$ connected, let

$$
x_{S, i, *}=\left(\frac{2}{r^{2}}\right)^{|S|} \text {. }
$$

We show that for $*, \# \in\{+,-\}$,

$$
\operatorname{Pr}\left(A_{S, i, *}\right)<x_{S, i, *} \prod_{(S, i, *) \sim(T, j, \#)}\left(1-x_{T, j, \#}\right),
$$

where $(S, i, *) \sim(T, j, \#)$ denotes adjacency of $A_{S, i, *}$ and $A_{T, j, \#}$ in the dependency graph of bad events i.e. $a^{*}(S) \cap a^{\#}(T) \neq \emptyset$. The theorem then follows from the general version of the local lemma, see for example Alon and Spencer [1].

It follows from Claim 2 that if $|S|=s$ then there are at most $k s(2 e r)^{t}$ events $A_{T, j \text {, \# }}$ with $|T|=t$ such that $(S, i, *) \sim(T, j, \#)$. Thus, using $1-x \geq e^{-2 x}$ for $0 \leq x \leq 1 / 2$ we have

$$
\begin{aligned}
x_{S, i, *} \prod_{(S, i, *) \sim(T, j, \#)}\left(1-x_{T, j, \#)}\right. & \geq\left(\frac{2}{r^{2}}\right)^{s} \prod_{t \geq 1}\left(1-\left(\frac{2}{r^{2}}\right)^{t}\right)^{k s(2 e r)^{t}} \\
& \geq\left(\frac{2}{r^{2}}\right)^{s} \exp \left\{-2 k s \sum_{t \geq 1}\left(\frac{4 e}{r}\right)^{t}\right\} \\
& =\left(\frac{2}{r^{2}}\right)^{s} \exp \left\{-\frac{8 k e s}{r-2 e}\right\} \\
& >\frac{1}{r^{2 s}},
\end{aligned}
$$

since for small values of $\epsilon$, the fact that $r / \ln r \geq 7 k \epsilon^{-2}$ implies

$$
r>2 e+\frac{8 k e}{\ln 2} \text {. }
$$

Thus (24) holds, proving the theorem.

\section{Splitting Algorithm}

In this section, we prove Theorem 4 .

Idea: We will define a sequence of sets $V=B_{1} \supseteq B_{2} \supseteq \cdots \supseteq B_{t}$ such that if $S \subseteq B_{j} \backslash B_{j+1}$ then $d_{i}^{+}(S), d_{i}^{-}(S)$ are large enough and further that every vertex in $B_{j} \backslash B_{j+1}$ has few neighbours in $B_{j+1}$. Then we will see that this latter condition accounts for the $-(\alpha+2 \epsilon) r$ term in the theorem. 
Assume we have $B \subseteq V$. Initially, $B=V$. We randomly colour the arcs of $D$ which are incident with $B$, with $k$ colours. Note that if $s_{0}=5 k \epsilon^{-2} \Phi^{-1} \ln n$

$$
\begin{aligned}
\operatorname{Pr}(\exists S \subseteq & \left.B, i \in[k] \text { s.t. }|S|>s_{0}, S \text { is connected and } \Phi_{i, S} \leq\left(1-\frac{\epsilon}{k}\right) \Phi\right) \\
& \leq 2 k n \sum_{s \geq s_{0}}(2 e r)^{s-1} e^{-\epsilon^{2} \Phi s /(2 k)} \leq 4 k n(2 e r)^{s_{0}} e^{-\epsilon^{2} \Phi s_{0} /(2 k)}=O\left(k n^{-1 / 5}\right) .
\end{aligned}
$$

So, in a sense the large sets, take care of themselves. Now consider the smaller sets. Let

$$
X_{0}=\left\{v: \exists S \subseteq B,|S| \leq s_{0}, S \text { is connected, } v \in S \text { and } i \in[k] \text { s.t. } \Phi_{i, S} \leq\left(1-\frac{\epsilon}{k}\right) \Phi\right\} .
$$

$X_{0}$ can be constructed in $O\left(n(e r)^{s_{0}}\right)=O\left(n^{2}\right)$ time.

$$
\mathbf{E}\left(\left|X_{0}\right|\right) \leq|B| \sum_{s=1}^{s_{0}}(2 e r)^{s-1} e^{-\epsilon^{2} \Phi s /(2 k)} \leq \frac{|B|}{2 e r}
$$

since $\Phi \geq 5 \epsilon^{-2} k \ln 2 e r$.

Therefore by Markov's Inequality,

$$
\operatorname{Pr}\left(\left|X_{0}\right| \geq \frac{|B|}{e r}\right) \leq \frac{1}{2}
$$

We repeat the above colouring until we find that $\left|X_{0}\right| \leq \frac{|B|}{e r}$. Now recursively define $X_{j}=X_{j-1} \cup\left\{v_{j}\right\}$ where $d^{+}\left(v_{j}, X_{j-1}\right) \geq(\alpha+\epsilon) r$ or $d^{-}\left(v_{j}, X_{j-1}\right) \geq(\alpha+\epsilon) r$, if such a $v_{j}$ exists. Now $|S| \leq \gamma n$ implies that $S$ contains at most

$$
\left(r|S|-d^{*}(S)\right) \leq \alpha r|S|
$$

arcs. Furthermore, $X_{j}$ has at least $(\alpha+\epsilon) r j$ arcs and at most $j+\frac{2|B|}{e r}$ vertices. Thus this process stops before $j$ reaches $\frac{\alpha|B|}{\epsilon e r}$, unless $\left|X_{j}\right|$ exceeds $\gamma n$ first. However, this latter possibility cannot happen since $\left|X_{0}\right|+\frac{\alpha|B|}{\epsilon e r} \leq\left(1+\frac{\alpha}{\epsilon}\right) \frac{1}{e r}|B| \leq \gamma|B| \leq \gamma n$ since $\gamma, \epsilon>$ $r^{-\frac{1}{2}}, \alpha<1$ implies $\gamma>\left(1+\frac{\alpha}{\epsilon}\right) \frac{1}{e r}$.

So if $X$ denotes $X_{j}$ when $v_{j+1}$ cannot be found, then

$$
|X| \leq \gamma|B|
$$

We will repeat the construction with $B$ replaced by $X$. Let $V=B_{1} \supseteq B_{2} \supseteq \cdots \supseteq B_{t}$ be the sequence of sets constructed. $B_{t}$ will be the first set of size at most $r^{-1} \ln n$. Since $\gamma<\frac{1}{2}$, we have $t \leq \log _{2} n$. Thus the expected number of re-colourings needed is at $\operatorname{most}^{2} \log _{2} n$ and is $\leq 3 \log _{2} n$ whp. We can "brute force" colour the arcs incident with $B_{t}$ so that every subset $S$ of $B_{t}$ satisfies $\Phi_{i, S} \geq\left(1-\frac{\epsilon}{k}\right) \Phi$. We use Theorem 3 to justify the success of this. The sequence of sets $B_{1}, B_{2}, \ldots, B_{t}$ satisfies 
- $\left|B_{j}\right| \leq \gamma^{j} n$.

- $S \subseteq B_{j} \backslash B_{j+1}$ implies $\Phi_{i, S} \geq\left(1-\frac{\epsilon}{k}\right) \Phi$.

- $v \in B_{j} \backslash B_{j+1}$ implies $v$ has at most $(\alpha+\epsilon) r j$ out-neighbours and at most $(\alpha+\epsilon) r j$ in-neighbours in $B_{j+1}$.

So if $S \subseteq V$ and $S_{j}=S \cap\left(B_{j} \backslash B_{j+1}\right)$,

$$
\begin{aligned}
d_{i}^{*}(S) & \geq \sum_{j=1}^{t-1}\left(d_{i}^{*}\left(S_{j}\right)-d^{*}\left(S_{j}, B_{j+1}\right)\right)+d_{i}^{*}\left(S_{t}\right) \\
& \geq \sum_{j=1}^{t-1}\left(1-\frac{\epsilon}{k} \Phi-(\alpha+\epsilon) r\right)\left|S_{j}\right|+\left(1-\frac{\epsilon}{k}\right) \Phi\left|S_{t}\right| \\
& \geq\left(1-\frac{\epsilon}{k} \Phi-(\alpha+\epsilon) r\right)|S| .
\end{aligned}
$$

This research was sponsored in part by National Science Foundation (NSF) grant no. CCR-0122581. 